\title{
Experiencia de la estrategia implementada por los equipos de respuesta rápida y seguimiento clínico para reducir la letalidad por COVID-19, Lambayeque-Perú 2020*
}

\section{Experience of the strategy implemented by the rapid response and clinical follow-up teams to reduce COVID-19 lethality, Lambayeque-Peru 2020*}

Cristian Díaz-Vélez ${ }^{1,2,5, a}$; Jorge Fernández-Mogollón ${ }^{2, a}$; Elizabeth Neciosup-Puicánº, ${ }^{2, b i n a ~}$ Colchado $^{2, \mathrm{c}}$;Jorge Ortiz-Millones ${ }^{2, \mathrm{~d}}$; Mileny Becerra-Torres ${ }^{2, \mathrm{e}}$; Natalie Zapata-Carrera ${ }^{2, \mathrm{f}}$; Roxana Neciosup-Puicán ${ }^{2, \mathrm{~b}}$; Yessica Neciosup-Liza ${ }^{2, \mathrm{~s}}$; Marco Manay-Chunga ${ }^{2, \mathrm{~h}}$; Antonio Sandoval-Larraín ${ }^{2, \mathrm{~h}}$;

Carlos Apolaya-Segura ${ }^{2, \mathrm{~h}}$; Jacqueline Betsabe Puicón-Suárez ${ }^{3,4, \mathrm{i}}$; Moisés Apolaya-Segura ${ }^{5,6, a}$

https://doi.org/10.35434/rcmhnaaa.2021.143.1285

\section{RESUMEN}

Introducción: El Perú fue considerado la mayor tasa de letalidad por COVID-19 (9.39\%); siendo Lambayeque en julio del 2020 presentó seroprevalencias más altas reportadas en el mundo para SARS-CoV-2 con $29,5 \%$. Objetivo. Describir la experiencia de la estrategia implementada por Equipos de Respuesta Rápida y Seguimiento Clínico de Casos de COVID-19 (ERSC) para reducir la letalidad por COVID-19 durante la primera ola en el 2020 Descripción de la estrategia. Se realizó una investigación operativa en una cohorte retrospectiva. La población estudiada estuvo conformada por los casos COVID-19 sospechosos y confirmados durante el periodo abril-diciembre 2020, atendidos en la Red Asistencial de Lambayeque. La metodología se llevó a cabo bajo la Implementación y funcionamiento de los ERSC que realizaban la Vigilancia Epidemiológica, rastreo y seguimiento clínico domiciliario de los casos sospechosos y confirmados de COVID-19. El proceso de captación y tamizaje inicial de los pacientes se realizó mediante tres procesos: tamizaje diferenciado, "call center" y vigilancia de rumores. Tras el tamizaje, se categorizaba al paciente en base a la atención que requería. Los casos eran asignados a los equipos de respuesta rápida quiénes acudían al domicilio del paciente para realizar las actividades indicadas de acuerdo al tipo de brigada. Las brigadas eran cuatro: brigadas de evaluación clínica domiciliaría, de diagnóstico o laboratorio a domicilio, de seguimiento clínico y brigada topo. c. La letalidad en pacientes moderados/severos disminuyó de 60\% (Semana Epidemiológica 19) a 10\% (Semana Epidemiológica 50) $(\mathrm{p}<0,001)$. La estrategia implementada y ejecutada redujo la letalidad por COVID-19 en población atendida.

Palabras Clave: COVID-19; pandemia; SARS-Cov-2; letalidad; epidemiología (Fuente: DeCS-BIREME).

\section{ABSTRACT}

Background: Peru was considered the highest fatality rate due to COVID-19 (9.39\%); Lambayeque being in July 2020 it presented the highest
FILIACIÓN

Facultad de Medicina, Universidad César Vallejo, Trujillo, Perú.

Hospital Nacional Almanzor Aguinaga Asenjo, EsSalud, Chiclayo, Perú.

. Faculad Medicina Universidad Pedro Ruiz Gallo, Lambayeque, Perú.

Sociedad Científica de Estudiantes de Medicina Humana de la Universidad Nacional Pedro Ruiz Gallo - SOCIEM UNPRG, Lambayeque, Perú.

. Instituto de Evaluación de Tecnologías en Salud e Investigación-IETSI, EsSalud, Lima Perú.

6. Centro de Investigación de Epidemiología Clínica y Medicina Basada en Evidencia (CECLIMBE), Universidad de San Martín de Porres, Lima, Perú.

Médico Epidemiólogo.

b. Licenciada en enfermería.

. Licenciada en Obstetricia.

Médico Cirujano.

Médico con especialidad en Terapia Física y Rehabilitación.

Medico con especialidad

Ingeniero en Sistemas.

Estudiante de Medicina Humana.

ORCID

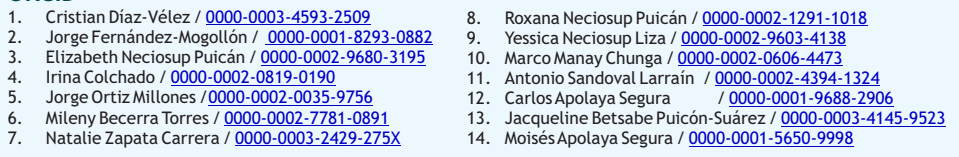

CORRESPONDENCIA

Cristian Díaz Vélez

EMAIL

cristian.diaz@essalud.gob.pe

CONFLICTOS DE INTERÉS

Los autores niegan conflictos de interés.

FINANCIAMIENTO

Autofinanciamiento.

*Ganadora del Tercer Puesto en el intercambio de experiencias, historias y buenas prácticas en Atención Primaria de Salud para enfrentar la pandemia de la COVID-19 otorgado por la Organización Panamericana de la Salud.

\section{AGRADECIMIENTOS}

A la Red Prestacional Lambayeque de EsSalud, la Gerencia Regional de Salud y el Comando Covid-19 de la Región Lambayeque.

REVISIÓN DE PARES

Recibido: $15 / 07 / 2021$

Aceptado: 01/09/2021

\section{COMO CITAR}

Díaz-Vélez, C., Fernández-Mogollón, J., Neciosup-Puicán, E., Colchado, I., Ortiz-Millones, J., Becerra-Torres, M. Zapata-Carrera, N., Neciosup-Puicán, R., Neciosup-Liza, Y., Manay-Chunga, M., Sandoval-Larraín, A., Apolaya-
Segura, C., Puicón-Suárez, J., \& Apolaya-Segura, M. Experiencia de la estrategia implementada por los equipos de resura, C., Puicon-Suarez, J., \& Apolaya-Segura, $M$. Experiencia de la estrategia implementada por los equipos de

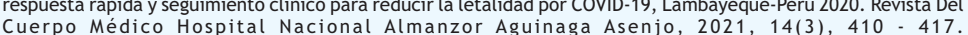
https://doi.org/10.35434/rcmhnaaa.2021.143.1285

\section{(c) (1)}

Esta obra está bajo una Licencia Creative Commons Atribución 4.0 Internacional.

Versión Impresa: ISSN: 2225-5109

Versión Electrónica: ISSN: 2227-4731

Cross Ref. DOI: $10.35434 / \mathrm{rcmhnaaa}$

OJS: https://cmhnaaa.org.pe/ojs 
seroprevalences reported in the world for SARS-CoV-2 with 29.5\%. Objective. To describe the experience of the strategy implemented by Rapid Response and Clinical Follow-up Teams of COVID-19 Cases (ERSC) to reduce COVID-19 lethality, during the first wave in 2020. Strategy description. Operational research was conducted in a retrospective cohort. The population studied was made up of suspected and confirmed COVID-19 cases during the April-December 2020 period, treated at the Lambayeque Healthcare Network. The methodology was carried out under the Implementation and operation of the ERSCs that performed Epidemiological Surveillance, tracking and home clinical follow-up of suspected and confirmed COVID-19 cases. The initial patient recruitment and screening process was carried out through three processes: differentiated screening, "call center" and rumor surveillance. After screening, the patient was categorized based on the care required. Cases were assigned to rapid response teams who went to the patient's home to carry out the activities indicated according to the type of brigade. There were four brigades: home clinical evaluation brigades, home diagnostic or laboratory brigades, clinical follow-up brigades and mole brigades. findings. Case fatality in moderate/severe patients decreased from 60\% (Epidemiological Week 19) to $10 \%$ (Epidemiological Week 50$)(p<0.001)$. The strategy implemented and executed reduced COVID-19 case-fatality in the population served.

Keywords: COVID-19; pandemic; SARS-Cov-2; fatality; epidemiology. (Source: DeCS-BIREME).

\section{INTRODUCCIÓN}

La pandemia por la enfermedad por coronavirus 2019, originada en Wuhan en el año 2019, se ha convertido en un desafío para los sistemas de salud que presentaban serias limitaciones en su funcionamiento ${ }^{(1)}$, por lo que múltiples países, han implementado diversas estrategias durante las etapas tempranas de la pandemia con el objetivo de disminuir la mortalidad y letalidad de su población ${ }^{(2)}$.

El Perú fue uno de los países con la mayor proporción de pobladores con COVID-19 en el mundo, considerándose como el país con la mayor tasa de letalidad $(9,39 \%)$ en el mundo, seguido de México $(9,0 \%)$, Hungría $(3,71 \%)$, Rumania $(3,17 \%)$ e Italia $(2,99 \%)^{(3,4)}$. En base a estos resultados, países alrededor del mundo iniciaron la gestión de nuevas estrategias para enfrentar la pandemia por COVID-19, tal es el caso de México, quién optó por plantear una estrategia de mitigación, tomando medidas con el objetivo de evitar que el sistema de salud sea extralimitado por un elevado número de demanda hospitalaria. Las medidas implementadas fueron: contar con el equipo y material necesario, con la infraestructura hospitalaria y personal de salud suficientes. Sin embargo, la letalidad por COVID-19 en México conservó una tendencia creciente hasta julio de 2020 , día en el que alcanzó un máximo de $12.40 \%{ }^{(5)}$.

La región de Lambayeque, situada en la costa norte del Perú, durante los tres primeros meses (Junio-Julio del 2020) de la pandemia por la COVID-19, presentó una seroprevalencia para Sars-CoV-2 de 29,5\% (IC95\% 27,6-31,5) considerada como una de las más altas reportadas en el mundo en ese período ${ }^{(6)}$. Un estudio realizado en 2 hospitales del Seguro Social de Salud (EsSalud) - Lambayeque, destinado para pacientes COVID 19 severos reportó una mortalidad de $60,2 \%$ en pacientes COVID-19 confirmado, falleciendo un aproximado de seis de cada 10 pacientes hospitalizados ${ }^{(7)}$. Sumado a esta elevada mortalidad, la región de Lambayeque registra una letalidad de $14,2 \%$, siendo la segunda más alta del país ${ }^{(8)}$.

En el Perú, con el objetivo de obtener una mejor vigilancia de casos COVID-19, en marzo del 2020 se aprobó la Directiva Sanitaria $N^{\circ}$ 088-MINSA-2020-CDC para la implementación y funcionamiento de los Equipos de Respuesta Rápida para realizar Vigilancia Epidemiológica de casos sospechosos de COVID-19 $9^{(9)}$. Posterior a este documento, EsSalud elaboró la guía de procedimientos para la vigilancia epidemiológica de casos sospechosos/confirmados por COVID-19(10). Debido a la elevada mortalidad y letalidad reportadas en la región Lambayeque, la guía antes citada fue modificada por la Red Asistencial Lambayeque EsSalud, desarrollando un plan de Implementación y funcionamiento de los Equipos de Respuesta y Seguimiento Clínico de Casos de COVID-19 (ERSC) que realizaban la Vigilancia Epidemiológica y seguimiento clínico domiciliario de los casos sospechosos y confirmados de COVID-19 ${ }^{(11)}$. En noviembre del 2020, el plan impulsado por la Red Asistencial Lambayeque EsSalud, recibió el reconocimiento de la Organización Panamericana de Salud ${ }^{(12)}$, siendo parte de los 12 finalistas del concurso de intercambio de experiencias, historias y buenas prácticas en atención primaria de la salud para enfrentar la pandemia de la COVID-19, evento en el que participaron instituciones a nivel La metodología implementada con el objetivo de detectar casos COVID-19 de manera temprana se fundamentó en la evidencia clínica de ese período, información que indicaba que el seguimiento precoz con la toma de pulsioximetría hospitalaria permitía detectar la "hipoxemia silenciosa" en los casos COVID-19 que no presentaban manifestaciones clínicas consideradas como graves. Estos pacientes no necesariamente tenían disnea, alteraciones de la frecuencia respiratoria o ruidos pulmonares patológicos, características que enmascaraban la hipoxia severa correspondiente a etapas avanzadas de la enfermedad ${ }^{(13,14)}$. En base a ello, estudios proponían a la pulsioxímetría prehospitalaria como un signo de alarma para la detección precoz de la hipoxemia silenciosa en pacientes COVID-19, estrategia que permitía actuar de manera inmediata con el traslado del paciente a un ambiente hospitalario y la administración de oxigenoterapia como primera medida terapéutica ${ }^{(13)}$. En relación al tratamiento, estudios demostraron que la administración temprana de dosis bajas de corticosteroides como la dexametasona reducía la mortalidad en pacientes COVID-19, siendo útil solo en los casos graves ${ }^{(15,16)}$.

El objetivo de este estudio fue determinar el efecto de la estrategia implementada por los equipos de respuesta rápida y seguimiento clínico de Casos de COVID-19 para reducir la letalidad por COVID-19, Lambayeque-Perú 2020.

\section{DESCRIPCIÓN DE LAESTRATEGIA}

Diseño y población de estudio

Se realizó una investigación operativa en una cohorte 
retrospectiva. La población de estudio estuvo conformada por todos los casos COVID-19 sospechosos y confirmados durante los meses de abril-diciembre 2020, que pertenecían a la Red Asistencial de Lambayeque. La metodología se llevó a cabo bajo la directiva de equipos de respuesta rápida de acuerdo al plan de Implementación y funcionamiento de los ERSC que realizaban la Vigilancia Epidemiológica y seguimiento clínico domiciliario de los casos sospechosos y confirmados de COVID-19 ${ }^{(10)}$.

La población de estudio fue de Lambayeque, región con una población estimada de 1197 260, ubicándose como la octava región más poblada del país con $4,1 \%$ de la población peruana total ${ }^{(17)}$. En diciembre del 2020 , esta región acumuló un total de 33,449 casos COVID-19 confirmados con un porcentaje de positividad del $21,7 \%$ y a 1,938 fallecidos $^{(8)}$. Del total de pacientes, la Red Asistencial Lambayeque de EsSalud ha reportado 21,104 casos COVID-19 confirmado, que representan el 63,09\% de pacientes COVID-19 confirmado de la región Lambayeque ${ }^{(18)}$. La modalidad de atención se realizó de manera ambulatoria y hospitalaria, registrándose 6,615 hospitalizaciones ${ }^{(19)}$. Se atendieron 43,245 pacientes y 245,627 seguimientos diarios entre abril-diciembre 2020 . (Tabla 2)

\section{Definición de variables}

La variable independiente fue la vigilancia epidemiológica mediante los Equipos de Respuesta Rápida y Seguimiento Clínico de Casos de COVID-19 (ERSC), esta estrategia involucra a cuatro tipos de brigadas que realizan la vigilancia epidemiológica y seguimiento clínico domiciliario de los casos sospechosos y confirmados de COVID-19 de acuerdo a su función designada: Brigada 1: evaluación clínica domiciliaría; Brigada 02: diagnóstico o laboratorio a domicilio ; Brigada 03: seguimiento clínico y Brigada 04: rescate ${ }^{(10)}$. Las brigadas brindaban atención de acuerdo a las recomendaciones clínicas para el manejo de pacientes con COVID-19 en los centros de atención y aislamiento temporal ${ }^{(20)}$ y las recomendaciones para el aislamiento domiciliario en contextos de pandemia por COVID-19 ${ }^{(21)}$.

La variable dependiente fue la letalidad en COVID-19, que se define como el cociente entre el número de personas fallecidas con diagnostico COVID-19 confirmado entre el número total de casos COVID-19 confirmados. Se consideró como caso confirmado a todo caso sospechoso o probable en el cual se obtuvo evidencia clínica, epidemiológica o de laboratorio, los casos se confirmaban por: prueba de laboratorio, nexo epidemiológico, o criterios clínicos con alto valor predictivo positivo ${ }^{(10)}$.

\section{Procedimientos}

Se realizó un plan estratégico de vigilancia epidemiológica en el primer nivel de atención en los establecimientos de salud que conforman la Red Asistencial Lambayeque, que brindan prestaciones de salud a la población asegurada del Seguro Social de Salud-EsSalud ${ }^{(10)}$.

El proceso de captación y tamizaje inicial de los pacientes se realizó mediante tres procesos: el primero corresponde al tamizaje diferenciado, cuando el paciente acude directamente a los establecimientos de salud de la Red Asistencial de Lambayeque y en base al triaje realizado se procedía a la apertura de la ficha epidemiológica; la segunda modalidad de captación se realizó mediante el "call center", en el que el paciente llamaba a los números telefónicos proporcionados por la red asistencial, se procedía a realizar un tamizaje epidemiológico vía telefónica y se aperturaba la ficha epidemiológica; finalmente el tercer proceso de captación se realizó mediante la vigilancia de rumores de pacientes con sospecha de ser caso COVID-19 que eran notificados a la Oficina de Inteligencia Sanitaria (OIS).

Los datos del paciente eran registrados por el personal de salud en el portal web de la Red asistencial de Lambayeque (http: //covid19ral.awp5.com/vistas/login2.html), y los médicos encargados de la vigilancia remota realizaban un tamizaje de riesgo evaluando al caso sospechoso de COVID-19 de acuerdo a su severidad y factores de riesgo. Si el paciente no presentaba sintomatología o factores de riesgo epidemiológico, se procedía a brindar orientación médica al paciente de acuerdo a sus necesidades. (Figura 1a.b.).
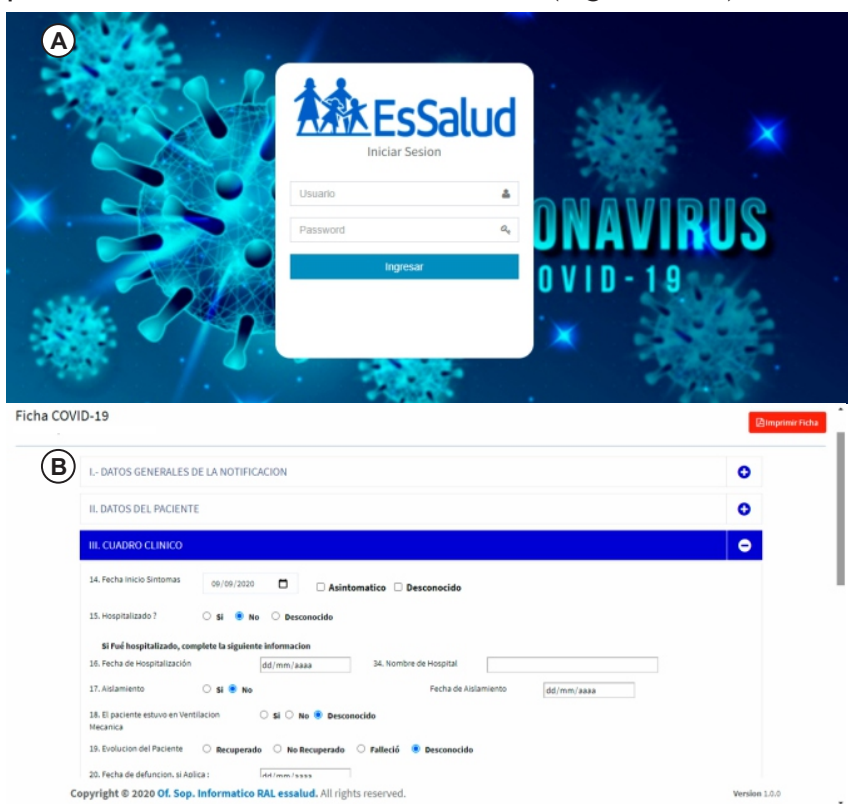

Figura 1

A. Portal web de vigilancia de pacientes COVID-19 sospechosos y confirmados. B. Ficha epidemiológica de pacientes COVID-19 registrados en el Portal web de la Red asistencial de Lambayeque. Enero - diciembre 2020

Tras el tamizaje, se categorizaba al paciente en base a la atención que requería, para ello se organizaban las brigadas de atención inmediata y evaluación clínica domiciliaría. Los casos eran asignados a los equipos de respuesta rápida quiénes acudían al domicilio del paciente para realizar las actividades indicadas de acuerdo al tipo de brigada.

\section{Formulación de las brigadas}

Las brigadas estaban constituidas de la siguiente manera:

Médico: Orientado a la atención de casos focalizados y a la búsqueda de los mismo, con la finalidad de brindar el tratamiento oportuno, educación y prevención en el marco de la COVID-19.

Biólogo: Orientado a la toma de muestra serológica, según 
indicación del médico de brigada y descartar casos positivos y negativos.

Psicólogo: Orientado al abordaje y soporte emocional oportuno al paciente ante la sospecha y/o caso positivo COVID-19 que resultaba de la visita domiciliaria con el biólogo, y cumpliendo así funciones presenciales y de telemonitoreo.

La coordinación de brigadas implementó y formuló las estrategias para la identificación y seguimiento de casos; según mapa geográfico se logró zonificar 14 rutas. (Tabla 1 ).

Tabla 1. Clasificación de acuerdo a zona de las 14 rutas de búsqueda de tratamiento y prevención de casos.

\begin{tabular}{ll}
\hline Ruta 1 & Chiclayo centro - Zona Hospital Naylamp \\
Ruta 2 & Zona de Pol. Chiclayo Oeste - Pimentel -San José \\
Ruta 3 & La Victoria \\
Ruta 4 & José Leonardo Ortiz \\
Ruta 5 & Tumán - Pomalca \\
Ruta 6 & Pátapo - Pucalá -Chongoyape \\
Ruta 7 & Reque - Eten - Pto Eten - Monsefú - Santa Rosa \\
Ruta 8 & Ferreñafe - Picsi - Pítipo \\
Ruta 9 & Lambayeque - Mochumí \\
Ruta 10 & Túcume - íllimo - Mórrope \\
Ruta 11 & Oyotún - Nueva Arica - Zaña - Cayaltí \\
Ruta 12 & Jayanca - Pacora -Salas \\
Ruta 13 & Motupe - Olmos \\
Ruta 14 & Chepén - Ucupe-Lagunas \\
\hline
\end{tabular}

La Oficina de Inteligencia Sanitaria (OIS) empleó 22 brigadas encargadas de la recolección de datos, elaboración de fichas epidemiológicas y evaluación, así como el seguimiento clínico de los pacientes sospechosos/confirmados por COVID19, las brigadas estaban clasificadas en cuatro grupos: brigadas de evaluación clínica domiciliaría, brigada de diagnóstico o laboratorio a domicilio, brigada de seguimiento clínico y la brigada de rescate también denominada brigada topo.

La brigada de evaluación clínica domiciliaría estaba integrada por un médico y un chofer para el traslado. El personal médico evaluaba al caso confirmado/sospechoso con factor de riesgo y determinaba el estado de severidad clínica del caso: asintomático, preasintomático, leve, moderado, severo o crítico ${ }^{(20)}$. De esta forma, establecía si el caso pasaba a monitoreo telefónico, a toma de muestra molecular/serológica o era necesario programar una próxima visita. De acuerdo a la evaluación se procedía a iniciar tratamiento de acuerdo a los protocolos o recomendaciones vigentes ${ }^{(22,23)}$ o coordinar el traslado al establecimiento de salud correspondiente. De ser necesario, generaba interconsultas a especialidades para teleconsultas.

La brigada de diagnóstico o laboratorio a domicilio estaba integrada por un biólogo y un chofer para el traslado. El personal de laboratorio realizaba la toma de muestra (después de 7 días de inicio de los síntomas, si el paciente era un caso severo se procedía a tomar la muestra inmediatamente). Finalmente registraba el resultado en la historia clínica electrónica del paciente.

La brigada de seguimiento clínico diario durante 14 días, estaba a cargo de médicos que realizaban la vigilancia de signos de alarma de manera remota, estos llamaban por teléfono a todos los pacientes captados. Los pacientes recibían soporte psicológico y nutricional, así como de medicina interna y medicina pediátrica de acuerdo a sus necesidades. Tras los 14 días de monitoreo, el médico encargado evaluaba todo el caso, si el paciente reportaba signos de alarma se informaba a la brigada de re-evaluación integrada por un médico y un psicólogo, quienes acudían al domicilio del paciente y determinaban la severidad clínica, de acuerdo a ello se sugería terapia de rehabilitación o el traslado a un centro de salud. Por el contrario, si el paciente refería una recuperación satisfactoria con sus funciones vitales estables se procedía a dar el alta. En caso sea necesario el médico expedía el certificado de buena salud y el personal de asistencia social tramitaba la documentación solicitada por el paciente. (Figura 3).

Al 15 de noviembre del 2020 , se atendió a un total de 46,279 casos mediante los equipos de respuesta rápida, 44,857 pacientes recibieron la alta epidemiologia siendo el $99 \%$ de los pacientes seguidos. El telemonitoreo fue realizado en 195,136 casos, además el número de pacientes que recibieron teleconsultas correspondientes al tamizaje inicial, psicología, nutrición, terapia física, o con trabajadoras sociales, fueron: 57,$133 ; 7,894 ; 7,841 ; 8,231$; 6,521 ; y 14,450 respectivamente.

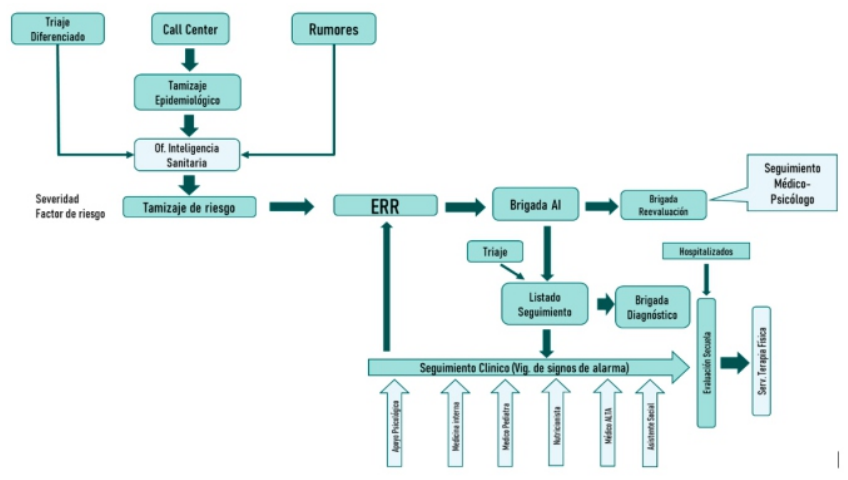

Figura 2.

Flujograma de procedimientos realizados por los equipos de respuesta rápida y seguimiento clínico de la red Asistencial Lambayeque.

Brigada de rescate (topo), son los equipos a los que se les destinó sectores específicos en lo que buscaban, indagaban e identificaban los casos sintomáticos según el mapa de calor (http://saludenlineaperu.com/MAPA_DE_CALOR) o rumores en los sectores designados, que permitían detectar posibles brotes de COVID-19. Tras su localización, se acudía con las brigadas y se realizaba un mapeo del área, solicitando en cada uno de los hogares del área de tamizaje información de pacientes con sintomatología compatible con la COVID-19. De acuerdo a los resultados obtenidos, se establecieron mapas de calor que eran actualizados constantemente. (Figura 3). 

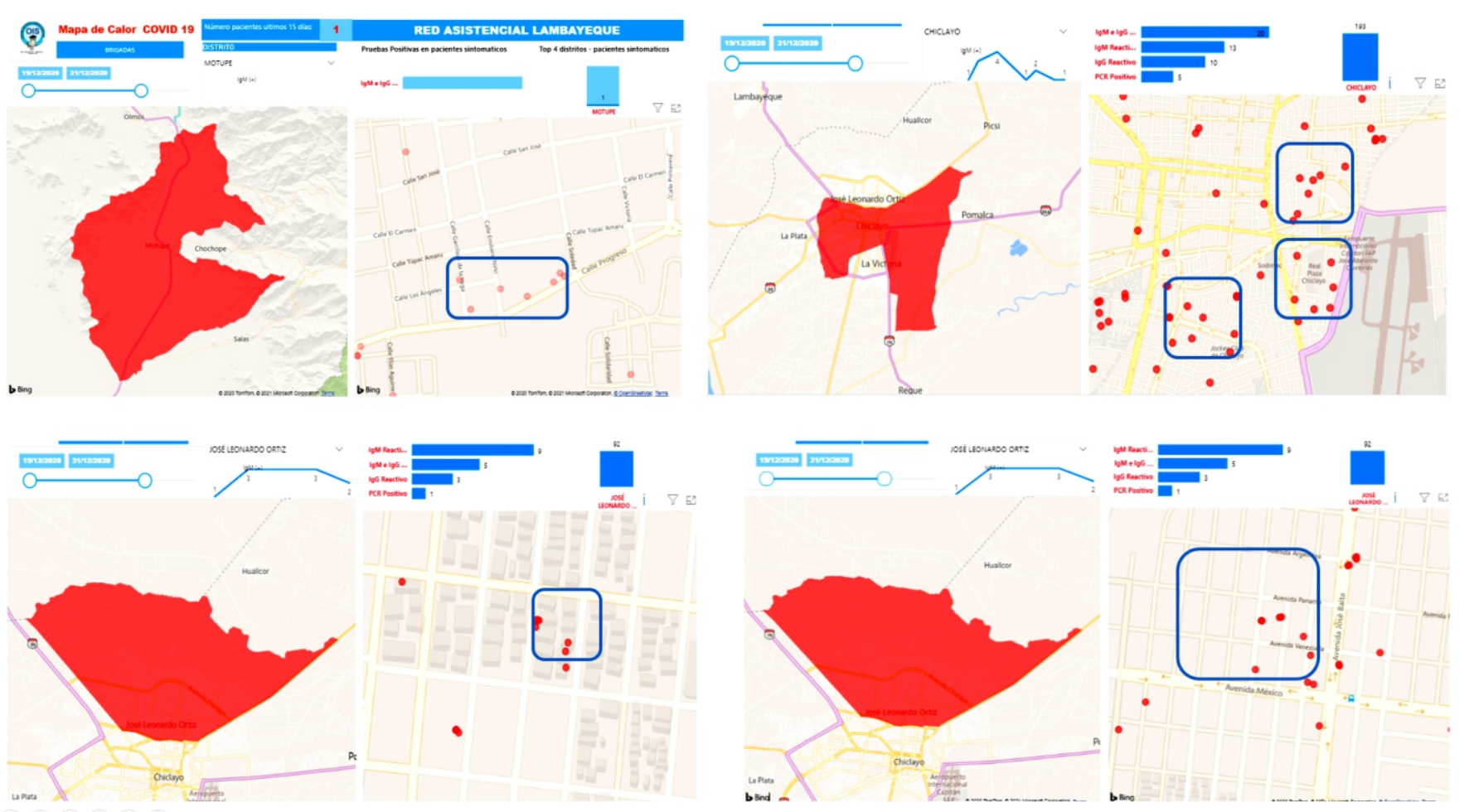

Figura 3.

Detección de brotes COVID-19 mediante Mapa de calor de la OIS-Red asistencial de Lambayeque. Enero-diciembre 2020.

\section{Desarrollo de Sala Situacional COVID 19 on-line:}

La información del sistema de vigilancia de COVID-19 fue elaborada de los documentos de la Oficina de Inteligencia Sanitaria, los formularios Power BI y la historia clínica electrónica de EsSalud, y para su visualización y actualización diaria se usó power Query, power pivot, power view. La sala situacional actual esta ubicada en: https://saludenlineaperu.com/salasituacional_ral

\section{Soporte psicológico}

Se reforzó las brigadas con psicólogos, quiénes tenían como función principal el acompañamiento al médico y/o biólogo que se encontraban focalizados en rutas en las diferentes partes de la región Lambayeque para realizar la atención psicológica oportuna y adecuada en cada uno de los pacientes y sus familias. De esta forma, el psicólogo cumplía la función de visitar el domicilio de los casos COVID-19 positivos, brindando atención personalizada que involucraba la concientización al paciente y sus familiares sobre los protocolos de cuidados a seguir en el marco de la COVID-19, finalmente ofrecía orientación psicológica con el objetivo de reducir o minimizar síntomas evitativos en el área emocional. Asimismo, cada psicólogo realiza la actividad de seguimiento de casos a través de la teleconsulta donde se comunica vía telefónica con el paciente en recuperación con tratamiento domiciliario o con el familiar responsable del paciente para seguir brindando el soporte emocional.

El soporte psicológico se implementó como una intervención necesaria, debido a que, en Lambayeque se presentó un elevado número de pacientes que se negaban a ser internados en hospitales a pesar de requerir hospitalización inmediata, solo 3 de cada 10 pacientes con indicación de hospitalización aceptaban ser hospitalizados, mientras que la cifra de pacientes que se rehusaban ascendía a 300 casos. La mayoría de estos pacientes señalaban tener miedo a morir en los hospitales ${ }^{(24)}$. Un estudio realizado a pacientes COVID19 positivos de la Red Asistencial de Lambayeque de Essalud durante el 2020, evaluó las características psicológicas y emocionales de 2,027 participantes, obteniendo como resultado que el enojo e inquietud presentaron la mayor prevalencia $(60.5 \%)$ independientemente de la frecuencia de presentación, mientras que la ansiedad fue el sentimiento más frecuente $(1,4 \%)^{(25)}$.

\section{Seroprevalencia en Lambayeque}

En la región de Lambayeque, durante el mes de mayo del 2020, se registró el mayor número de defunciones debido a la COVID-19, superando las cifras alcanzadas en meses anteriores ${ }^{(8)}$; de esta manera, se evidenció que Lambayeque era una de las ciudades más afectadas en el Perú a causa de la pandemia. Con el objetivo de evaluar la extensión de la afectación de esta región, durante los meses de junio a julio del 2020 se llevó a cabo un estudio de seroprevalencia para SARS-CoV-2 con población de la región de Lambayeque. De las 2,010 unidades de estudio se obtuvo una seroprevalencia de 29,5\% (IC 95\% 27,6-31,5), siendo similar a la de las tres provincias: Lambayeque, Ferreñafe y Chiclayo $(32,9,22,5$ y 29,2 respectivamente). Los hallazgos determinaron que la región de Lambayeque tenía la mayor seroprevalencia para SARS-CoV-2 a nivel mundial, superando a Delhi, India $(23,48 \%)^{(6)}$.

Durante el mismo período (junio-julio) se realizó un estudio de seroprevalencia para SARS-CoV-2 en Lima, obteniendo un resultado de $20,8 \%$ (IC del 95\%: 17,2-23,5) ${ }^{(26)}$, cifra inferior a 
la obtenida en Lambayeque. Trascurridos 4 meses del inicio de la pandemia en Perú y un mes después de la ejecución de los estudios citados previamente, se realizó un estudio de seroprevalencia para SARS-CoV-2 en la ciudad de Iquitos y tras un mes de seguimiento se obtuvo una seroprevalencia ajustada del 66\% (IC del 95\%: 62-70) ${ }^{(27)}$, esta cifra elevada en comparación a Lambayeque fue a consecuencia de la variación temporal, ya que al transcurrir un mayor tiempo la prevalencia de la enfermedad COVID-19 aumentó.

\section{Implementación de la estrategia: "Luchando contra el Covid silencioso"}

En la semana epidemiológica número 25 de la red Asistencial de Lambayeque se implementó la estrategia "Luchando contra el Covid silencioso" ${ }^{(28)}$ para la detección temprana de COVID-19 en pacientes sin sintomatología, pero con hipoxemia silenciosa (saturación de oxígeno inferior a 94\%). Se realizaron campañas en donde los agentes comunitarios, denominados promotores de salud, fueron capacitados para el uso del pulsioxímetro de pulso con el objetivo de captar posibles casos de COVID-19, se evaluó la saturación de oxígeno y frecuencia cardiaca.

Este programa se desarrolló en 5 campañas en los distritos de Motupe, Lambayeque, Monsefú, Tumán y Chiclayo; se captó a un total de 18506 pacientes, un porcentaje de pacientes que presentaban factores de riesgo y/o comorbilidades ingresaron a un grupo de monitoreo diario. De este grupo de pacientes se desprendió los casos catalogados como alarma, quienes presentaron saturación de oxígeno inferior a 94\%, de acuerdo a su evolución clínica se les indicaba hospitalización o continuar con el monitoreo domiciliario. En julio del 2020, esta estrategia obtuvo el reconocimiento de la ministra de defensa del Perú, quién señaló que este plan, diseñado e implementado en la región de Lambayeque, era uno de los programas más interesantes realizados a nivel nacional, siendo un modelo replicable y con capacidad de adaptación para cada comunidad ${ }^{(29)}$.

\section{Cumplimiento del protocolo de atención}

Para verificar que los equipos de respuesta rápida y seguimiento clínico cumplían con los protocolos de atención establecidos durante la pandemia COVID-19, el personal de enfermería de la OIS aplicó un cuestionario a los pacientes mediante llamada telefónica, en donde se formulaban preguntas que evaluaban el cumplimiento del protocolo de atención por parte del personal de salud. Tras la evaluación de los resultados obtenidos durante julio a diciembre del 2020, se obtuvo que los equipos de respuesta rápida y seguimiento clínico sí cumplían con el protocolo de atención. Se evaluaron tres áreas: tamizaje inicial, evaluación clínica y telemonitoreo, obteniéndose cifras de cumplimiento superiores a $78 \%, 80 \%$ y $79 \%$ respectivamente. Adicionalmente, el $70 \%$ de los pacientes refirieron una elevada satisfacción del servicio brindado por los equipos de respuesta rápida y seguimiento clínico con puntuaciones superiores a 8 puntos en una escala de evaluación del 1 al $10^{(30)}$.

\section{HALLAZGOS}

\section{Características de la población}

Se atendieron 43245 pacientes y 245627 seguimientos diarios entre abril-diciembre 2020.

Tabla 2. Características de los pacientes COVID-19 de la Red Asistencial de Lambayeque confirmados durante los meses de abril-diciembre 2020.

\begin{tabular}{lcc} 
Características & $\mathrm{n}=43245$ & $\%$ \\
Edad (años) & $46.72(46)$ & $\pm 20.04(32-62)$ \\
Sexo & & \\
\multicolumn{1}{c}{ Femenino } & 25125 & 58.1 \\
$\quad$ Masculino & 18120 & 41.9 \\
Asintomáticos & 8545 & 19.76 \\
Aislamiento & 4065 & 9.4 \\
Hospitalización & 10392 & 24.03 \\
\hline
\end{tabular}

El porcentaje de pacientes Sars-CoV-2 fallecidos en su domicilio o en las primeras 24 horas de hospitalización de la Red Asistencial de Lambayeque, en la semana epidemiológica 15 presentó una elevada frecuencia de letalidad, en la que 1 de cada 3 pacientes moría. Sin embargo, tras la implementación de los equipos de respuesta rápida la frecuencia de pacientes fallecidos disminuyó significativamente durante las semanas siguientes, presentando un promedio de letalidad del $2 \%$ durante las semanas epidemiológicas 37 a 46, y las semanas posteriores no se presentaron defunciones. (Gráfico 1 ).

Gráfico 1. Pacientes Sars-CoV-2 fallecidos en su domicilio o en las primeras 24 horas de hospitalización de la Red Asistencial de Lambayeque durante los meses de abrildiciembre 2020.

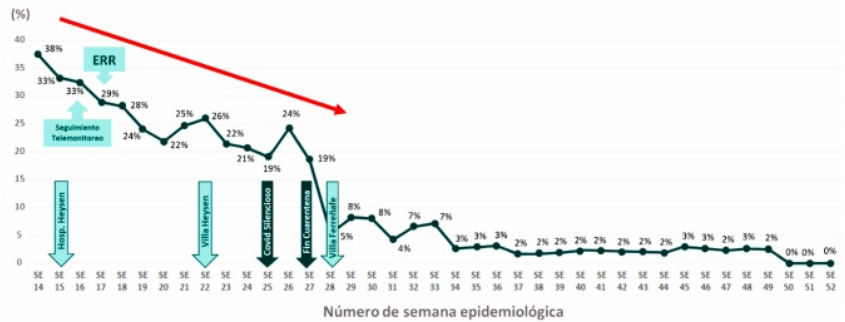

Se observó que en semanas previas a la implementación de los equipos de respuesta rápida más del $50 \%$ de pacientes Sars-CoV-2 referidos como moderados/severos tenían un desenlace fatal, mientras que 4 semanas después de la implementación de los equipos de respuesta rápida la mortalidad disminuyó al $10 \%$ y las semanas posteriores presentó frecuencias regulares entre el 10 a 35\% con una tendencia a disminuir la frecuencia de letalidad. (Gráfico 2). 
Gráfico 2. Frecuencia de pacientes Sars-CoV-2 de la Red Asistencial de Lambayeque referidos como moderados/severos que fallecieron durante los meses de abril-diciembre 2020.

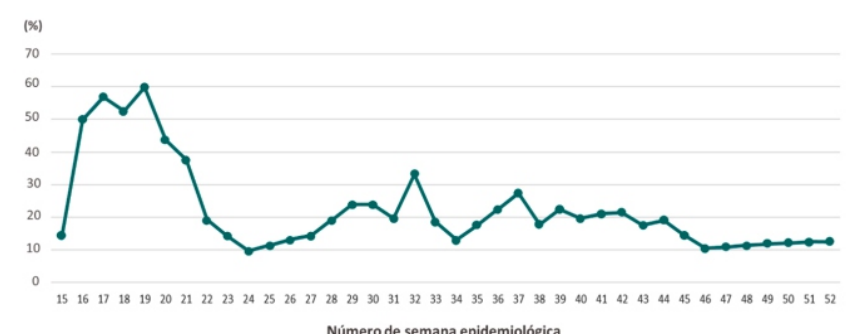

En relación a la letalidad de pacientes Sars-CoV-2 según el seguimiento clínico, los pacientes que no tuvieron seguimiento presentaron una elevada frecuencia de letalidad con una frecuencia de 25 a $40 \%$, mientras que los pacientes que recibieron seguimiento clínico presentaron una frecuencia de letalidad inferior al 25 a 20\%. (Gráfico 3).

Gráfico 3. Mortalidad de pacientes Sars-CoV-2 según seguimiento clínico de la Red Asistencial de Lambayeque durante los meses de abril-diciembre 2020.

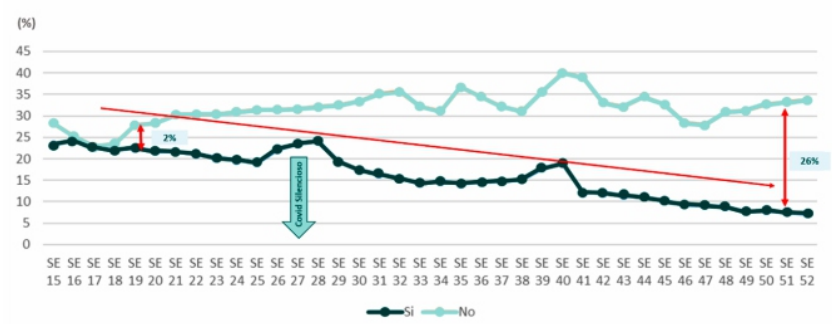

Respecto al tiempo de enfermedad al momento del ingreso a hospitalización en pacientes SARS-CoV-2 según seguimiento clínico, en el mes de mayo se presentó un promedio de 10.8 días de tiempo de enfermedad al momento del ingreso a hospitalización, de una forma coincidente, semanas después tras la implementación de los equipos de respuesta rápida presentó una tendencia a disminuir el promedio de tiempo hasta alcanzar valores de 5.4 días en el mes de diciembre. (Gráfico 4).

Gráfico 4. Tiempo de enfermedad al momento del ingreso a hospitalización en pacientes Sars-CoV-2 según seguimiento clínico de la Red Asistencial de Lambayeque durante los meses de abril-diciembre 2020.

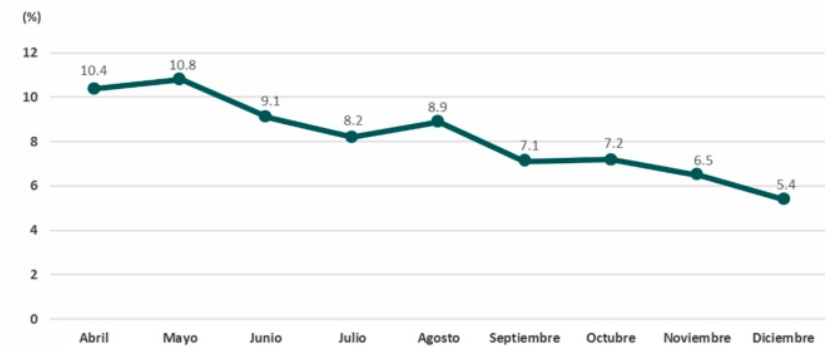

En relación a la estrategia, "Luchando contra el Covid silencioso", se captó a un total de 18506 pacientes, 1345 casos ingresaron a un monitoreo diario por tener comorbilidades o factores de riesgo, se presentaron 231 alarmas y 14 de ellos ingresaron a hospitalización donde solo 3 pacientes fallecieron, presentándose una letalidad del $0.22 \%$. (Figura 5 ).

Figura 5. Flujograma de la estrategia "Luchando contra el Covid silencioso". Enero-diciembre 2020.

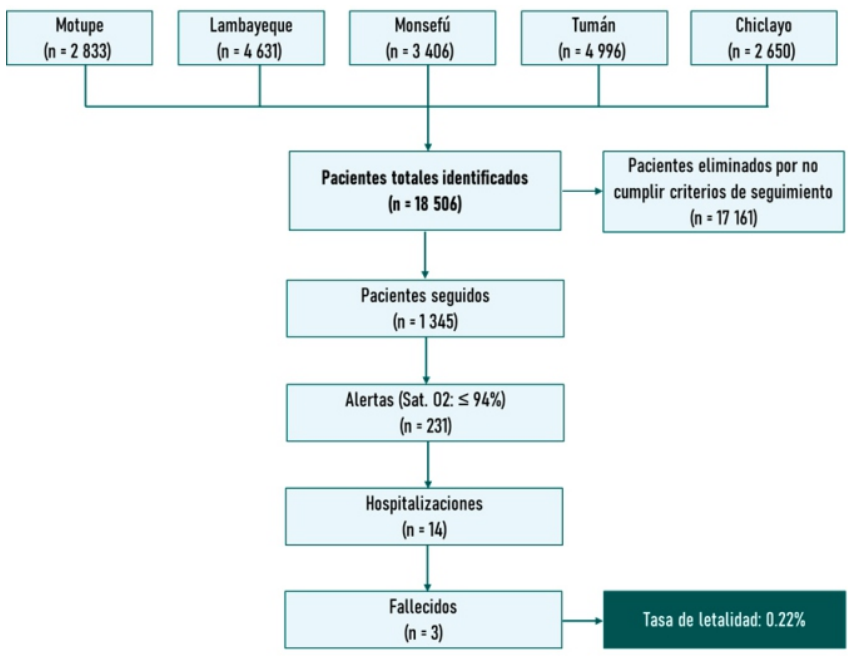

\section{Lecciones aprendidas:}

- La detección temprana de casos redujo la mortalidad y tiempo de hospitalización.

- El seguimiento de casos considerando espacio y tiempo utilizando herramientas informáticas disponible a tiempo real permite tomar acciones oportunas para el control de brotes.

- $\quad$ El uso eficiente del personal de salud para vigilancia epidemiológica e intervención epidemiológica.

\section{CONCLUSIONES}

La implementación de los ERR y SC, se realizó con modificaciones en su estructura y funcionamiento en concordancia con las estrategias implementadas, mostrando un impacto no sólo el uso eficiente de los recursos disponibles en momento que el personal de salud era escaso, además de implementación de los ERR y SC muestra un impacto en la reducción de la letalidad de casos covid-19 durante el 2020.

\section{REFERENCIAS BIBLIOGRÁFICAS}

1. Cabezas C. Pandemia de la COVID-19: Tormentas y retos. Rev Peru Med Exp Salud Publica. 2020;37(4):603-4. Doi: 10.17843/rpmesp.2020.374.6866

2. Organización Mundial de la Salud. Actualización de la estrategia frente a la COVID-19 [Internet]. Ginebra: Organización Mundial de la Salud, OMS; 2020 [Citado el 20 de setiembre del 2021]. Disponible en: https: / / www. who.int/docs/default-source/coronaviruse/covidstrategy-update-14april2020 es.pdf

3. Ministerio de Salud. Situación Actual COVID-19, 02 de Junio [Internet]. Lima: Centro Nacional de Epidemiología, Prevención y Control de Enfermedades, MINSA; 2020 [Citado el 20 de setiembre del $\left.\begin{array}{lllll}2 & 0 & 2 & 1\end{array}\right]$. D is pon i b l e e n : https://www.dge.gob.pe/portal/docs/tools/coronavirus/coronavir us020621.pdf

4. Pinzón JED. Letalidad por SARS-COV-2 a nivel mundial. Repert med c i r. $2021 ; 30$ ( S u pl. Nú m.1):84-88. Do i : 10.31260/RepertMedCir.01217372.1255 
5. Del Río Monges J. Estrategia de mitigación para enfrentar al COVID-19 en México: Análisis y resultados tras un año de pandemia [Internet]. Ciudad de México: Dirección General de Finanzas, Instituto Belisario Domínguez; 2021 [Citado el 25 de setiembre del 2021]. Disponible en: http: / /bibliodigitalibd.senado.gob.mx/handle/123456789/5160.

6. Díaz-Vélez C, Failoc-Rojas VE, Valladares-Garrido MJ, Colchado J, Carrera-Acosta L, Becerra M, et al. SARS-CoV-2 seroprevalence study in Lambayeque, Peru. June-July 2020. PeerJ. 2021;9:e11210. Doi: $10.7717 /$ peerj. 11210

7. Díaz-Vélez C, Urrunaga-Pastor D, Romero-Cerdán A, Peña-Sánchez E, Fernández Mogollon J, Cossio Chafloque J, et al. Risk factors for mortality in hospitalized patients with COVID-19 from three hospitals in Peru: a retrospective cohort study [version 1; peer review: 2 approved]. F1000Research. 2021;10(224). Doi: 10.12688/f1000research.51474.1

8. Ministerio de Salud. Sala Situacional COVID-19 [Internet]. Lima: Instituto Nacional de Salud y Centro Nacional de Epidemiologia, Prevención y Control de Enfermedades, MINSA; 2020. [Citado el 30 de jun io del 20021 ]. Dis pon ible en: https://covid19.minsa.gob.pe/sala_situacional.asp

9. Ministerio de Salud. Directiva Sanitaria para la implementación y funcionamiento de los Equipos de Respuesta Rápida (ERR) que realizan la Vigilancia Epidemiológica de casos sospechosos de COVID19 [Internet]. Lima: Centro Nacional de Epidemiologia, Prevención y Control de Enfermedades, MINSA; 2020. [Citado el 30 de junio del 20021 ]. D is pon i b l e n https://cdn.www.gob.pe/uploads/document/file/574546/RM_1412020-MINSA CON_ANEXOS 1.PDF

10. Red Asistencial Lambayeque, EsSalud. Guía de procedimientos para a vigilancia epidemiológica de casos sospechosos/confirmados por COVID-19 [Internet]. Chiclayo: Oficina de Gestión de Calidad y Control Interno, RAL-EsSalud. [Citado el 30 de junio del 2021]. Disponible en: www.essalud.gob.pe

11. Red Asistencial Lambayeque, EsSalud. Implementación y funcionamiento de los Equipos de Respuesta y Seguimiento Clínico de Casos de COVID-19 (ERSC) que realizaban la Vigilancia Epidemiológica y seguimiento clínico domiciliario de los casos sospechosos y confirmados de COVID-19. [Internet]. Chiclayo: Oficina de Gestión de Calidad y Control Interno, RAL-EsSalud. [Citado el 30 de junio del 2021]. Disponible en: www.essalud.gob.pe

12. Organización Panamericana de la Salud. Intercambio de experiencias, historias y buenas prácticas en Atención Primaria de Salud para enfrentar la pandemia de la COVID-19 [Internet]. Washington: Oficina Regional para las Américas de la Organización Mundial de la Salud, OMS; 2020 [Citado el 03 de agosto del 2021]. Disponible en: https://www. paho.org/es/peru/intercambioexperiencias-historias-buenas-practicas-atencion-primaria-saludpara-enfrentar

13. Jouffroy R, Jost D, Prunet B. Prehospital pulse oximetry: a red flag for early detection of silent hypoxemia in COVID-19 patients. Crit Care. 2020;24(1):313. Doi: 10.1186/s13054-020-03036-9

14. Ottestad W, Seim M, Mæhlen JO. COVID-19 with silent hypoxemia. Tidsskr Nor Laegeforen. 2020;140(7). Doi: 10.4045/tidsskr.20.0299.

15. Deng CX. Glucocorticoids save lives in COVID-19 patients. Int J Biol Sci. 2020; 16 (13): 2477-2478. Doi: 10.7150 / ijbs.49125

16. Rafiullah $M$, Siddiqui K. Corticosteroid use in viral pneumonia: experience so far and the dexamethasone breakthrough in coronavirus disease-2019. J Comp Eff Res. 2020;9(18):1247-1254. Doi: $10.2217 /$ cer-2020-0146.

17. Instituto Nacional de Estadística e Informática. Lambayeque, resultados definitivos [Internet]. Lima: Instituto Nacional de Estadística e Informática, INEI; 2018 [Citado el 03 de agosto del $\left.\begin{array}{lllll}2 & 0 & 2 & 1\end{array}\right]$. D is po n b l e e n https://www.inei.gob.pe/media/MenuRecursivo/publicaciones_dig itales/Est/Lib1560/14TOMO_01.pdf

18. Ministerio de Salud. Situación Actual COVID-19, 31 de Diciembre [Internet]. Lima: Centro Nacional de Epidemiología, Prevención y Control de Enfermedades, MINSA; 2020 [Citado el 20 de setiembre del $\left.\begin{array}{llllllll}2 & 0 & 2 & 1\end{array}\right]$. D i s p o n i b l e $n$ : https://www.dge.gob.pe/portal/docs/tools/coronavirus/coronavir us311220.pdf

19. Red Asistencial Lambayeque, EsSalud. Sala Situacional COVID-19 [Internet]. Chiclayo: Oficina de Gestión de Calidad y Control Interno, RAL-EsSalud; 2020. [Citado el 24 de setiembre del 2021]. Disponible en: http://redasistenciallam.com/salasituacional_ra

20. Instituto de Evaluación de Tecnologías en Salud e Investigación, EsSalud. Recomendaciones clínicas para el manejo de pacientes con COVID-19 en los centros de atención y aislamiento temporal [Internet]. Lima: Instituto de Evaluación de Tecnologías en Salud e Investigación, EsSalud; 2021 [Citado el 25 de setiembre del 2021]. D i s p o n b l e http://www.essalud.gob.pe/ietsi/pdfs/guias/reportes/R ecomenda ciones manejo de COVID19 en CAAT Mayo2021.pdf

21. Instituto de Evaluación de Tecnologías en Salud e Investigación, EsSalud. Recomendaciones para el aislamiento domiciliario en contextos de pandemia por COVID-19 [Internet]. Lima: Instituto de Evaluación de Tecnologías en Salud e Investigación, EsSalud; 2020 [Citado el 25 de setiembre del 2021]. Disponible en: http://www.essalud.gob.pe/ietsi/pdfs/guias/Recomendaciones ai slamiento_domiciliario_COVID_19.pdf

22. Ministerio de Salud. Prevención, Diagnóstico y Tratamiento de personas afectadas por COVID-19 en el Perú. [Internet]. Lima: Centro Nacional de Epidemiologia, Prevención y Control de Enfermedades, MINSA; 2020. [Citado el 24 de setiembre del 2021]. Disponible en: https://cdn.www.gob.pe/uploads/document/file/582550/ANEXO_RM_193-2020-MINSA.PDF

23. Ministerio de Salud. Manejo ambulatorio de personas afectadas por la COVID-19 en el Perú. [Internet]. Lima: Centro Nacional de Epidemiologia, Prevención y Control de Enfermedades, MINSA; 2020. [Citado el 24 de setiembre del 2021]. Disponible en: $\mathrm{h} \mathrm{t} \mathrm{t} \mathrm{ps}$ : / / portal. i n e n s s d . p e / w p content/uploads/2020/11/Resoluci\%C3\%B3n-MinisterialN\%C2\%B0947-2020-MINSA.pdf

24. Polo P. (2020) Pacientes con la COVID-19 se niegan a hospitalizarse en Lambayeque Diario Correo [Internet] 10 de octubre del 2020. D i s p o n ible

e $n$ https: / / diariocorreo.pe/edicion/lambayeque/lambayequepacientes-con-la-covid-19-se-niegan-a-hospitalizarsenoticia/?ref=dcr

25. Quesquén Chavesta MN, Ordoñez Ramírez LE. Características de la evaluación psicológica realizada a los casos de SARS-COV-2 en la red asistencial de Lambayeque, durante el estado de emergencia sanitaria 2020 [Tesis]. Lambayeque: Facultad de Medicina Humana, Universidad Señor de Sipán; 2021 [Citado el 06 de noviembre del 2021]. Disponible en: https: / /bit.ly/3Hk6xzh

26. Reyes-Vega MF, Soto-Cabezas MG, Cárdenas F, Martel KS, Valle A, Valverde J, et al. SARS-CoV-2 prevalence associated to low socioeconomic status and overcrowding in an LMIC megacity: A population-based seroepidemiological survey in Lima, Peru. E C I in i c a lMedicine. 2021;34:100801. Doi : 10.1016/j.eclinm.2021.100801

27. Álvarez-Antonio C, Meza-Sánchez G, Calampa C, Casanova W, Carey C, Alava F, et al. Seroprevalence of anti-SARS-CoV-2 antibodies in Iquitos, Peru in July and August, 2020: a population-based study. The Lancet Global Health. 2021;9(7):e925-e31. Doi: 10.1016/S2214109X(21)00173-X

28. Establecimiento de Salud Jorge Chávez Geresa, Red Asistencial Lambayeque, EsSalud. Lucha contra el COVID silencioso [Internet]. Lambayeque: Red Asistencial Lambayeque, EsSalud; 2020. [Citado el 24 de setiembre del 2021 ]. Disponible en: https: / / www.paho.org/es/documentos/lucha-contra-covidsilencioso.

29. TVPerú Noticias. Ministra Esparch: "El Plan Sipán es uno de los programas más interesantes que se ha hecho a nivel nacional, y que surgió aquí en esta región, a partir de una necesidad de la propia población" [Tweet]. Twitter. 17 de junio del 2021. [Citado el 4 de

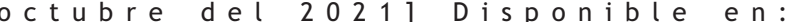
https://twitter.com/noticias_tvperu/status/1405550663690600448 ?s=12\&fbclid=IwAR3ssYGRf4TQwPoD1i31spxnjHakXzlBGFPYkRhpM2p 1 MOBW1JfRaCjG4U.

30. Huamán Benites ZR, Urbina Diaz K. Evaluación del cumplimiento del protocolo de atención de los equipos de respuesta rápida y seguimiento clínico durante la pandemia por Sarscov-2, ESSALUD Lambayeque, julio-diciembre 2020. [Tesis]. Lambayeque: Facultad de Medicina Humana, Universidad Señor de Sipán; 2021 [Citado el 06 de noviembre del 2021]. Disponible en: https: / /bit.ly/2YLqIVC. 